\title{
SOME PLANAR MONOMIALS IN CHARACTERISTIC 2
}

\author{
ZACHARY SCHERR AND MICHAEL E. ZIEVE
}

\begin{abstract}
Planar functions over finite fields give rise to finite projective planes and other combinatorial objects. They were originally defined only in odd characteristic, but recently Zhou introduced a definition in even characteristic which yields similar applications. In this paper we show that certain functions over $\mathbb{F}_{2^{r}}$ are planar, which proves a conjecture of Schmidt and Zhou. The key to our proof is a new result about the $\mathbb{F}_{q^{3}}$-rational points on the curve $x^{q-1}+y^{q-1}=z^{q-1}$.
\end{abstract}

\section{INTRODUCTION}

Let $q=p^{r}$ where $p$ is prime and $r$ is a positive integer. If $p$ is odd then a planar function on $\mathbb{F}_{q}$ is a function $F: \mathbb{F}_{q} \rightarrow \mathbb{F}_{q}$ such that, for every $d \in \mathbb{F}_{q}^{*}$, the function $c \mapsto F(c+d)-F(c)$ is a bijection on $\mathbb{F}_{q}$. Planar functions have been used to construct finite projective planes [4], relative difference sets [5], error-correcting codes [1, and $S$-boxes in block ciphers with optimal resistance to differential cryptanalysis [7].

If $p=2$ then there are no functions $F: \mathbb{F}_{q} \rightarrow \mathbb{F}_{q}$ satisfying the defining property of a planar function, since 0 and $d$ have the same image as one another under the map $c \mapsto F(c+d)-F(c)$. Recently Zhou [10] introduced a characteristic 2 analogue of planar functions, which have the same types of applications as do odd-characteristic planar functions. These will be the focus of the present paper. If $p=2$, we say that a function $F: \mathbb{F}_{q} \rightarrow \mathbb{F}_{q}$ is planar if, for every $d \in \mathbb{F}_{q}^{*}$, the function $c \mapsto F(c+d)+F(c)+d c$ is a bijection on $\mathbb{F}_{q}$. Schmidt and Zhou [8, 10] showed that any function satisfying this definition can be used to produce a relative difference set with parameters $(q, q, q, 1)$, a finite projective plane, and certain codes with unusual properties. In what follows, whenever we refer to a planar function in characteristic 2, we mean a function satisfying Zhou's definition.

In the recent paper 8, Schmidt and Zhou studied planar functions in characteristic 2 which have the form $c \mapsto a c^{t}$, where $a \in \mathbb{F}_{q}^{*}$. They exhibited two classes of such planar monomials on $\mathbb{F}_{q}$ where $q=2^{r}$ :

- $t=2^{k}$ for any $k \geq 0$, any $r$, and any $a \in \mathbb{F}_{q}^{*}$

- $t=2^{k}+1$ where $r=2 k$ and $a=b^{2}+b$ for any $b \in \mathbb{F}_{2^{k}} \backslash \mathbb{F}_{2}$.

2010 Mathematics Subject Classification. 51E20, 11G20, 11 T06.

The authors were partially supported by NSF grant DMS-1162181. 
They also conjectured [8, Conj. 7] that, when $r=6 k$ and $t=4^{k}+16^{k}$, there exists $a \in \mathbb{F}_{q}^{*}$ such that $c \mapsto a c^{t}$ is planar on $\mathbb{F}_{2^{r}}$. We will prove this conjecture in the following more precise form:

Theorem 1.1. For any positive integer $k$, write $Q=4^{k}$ and $q=Q^{3}$. If $a \in \mathbb{F}_{q}^{*}$ is a $(Q-1)$-th power but not a $3(Q-1)$-th power, then the function $c \mapsto a c^{Q^{2}+Q}$ is planar on $\mathbb{F}_{q}$.

Our proof relies on the following result of independent interest:

Theorem 1.2. If $Q>1$ is a power of 2 , and $u, v \in \mathbb{F}_{Q^{3}}^{*}$ satisfy $u^{Q-1}+$ $v^{Q-1}=1$, then uv is a cube in $\mathbb{F}_{Q^{3}}$.

We note that, in subsequent work, Voloch and the second author [9] have proved an analogous result for odd prime powers.

We conclude this introduction with some general remarks about planar monomials in characteristic 2. Since $c \mapsto a c^{t}$ is planar on $\mathbb{F}_{q}$ if and only if $c \mapsto a c^{t+q-1}$ is planar, we will restrict to planar monomials of degree less than $q$. All known planar monomials over $\mathbb{F}_{2^{r}}$ of degree between 2 and $2^{r}-1$ have degree of the form $2^{i}+2^{j}$. In light of this, we divide the classification of planar monomials in characteristic 2 into two parts:

- monomials of degree $2^{i}+2^{j}$, and

- monomials of other degrees.

We checked via computer that, for $r \leq 14$, every planar monomial over $\mathbb{F}_{2^{r}}$ of degree between 2 and $2^{r}-1$ has degree $2^{i}+2^{j}$. We suspect that all planar monomials over $\mathbb{F}_{2^{r}}$ of degree $2^{i}+2^{j}$ with $0 \leq i<j<r$ are displayed in Theorem 1.1 and Proposition 3.2, but we cannot prove this. We will present results in this direction in Section 3, after proving our main results in the next section.

\section{Proof of Theorem 1.1}

In this section we prove Theorem 1.1, Our proof relies on Theorem 1.2, which we prove first.

Proof of Theorem 1.2. If $Q=2^{r}$ where $r$ is odd, then $Q^{3}-1 \equiv 1(\bmod 3)$, so every element of $\mathbb{F}_{Q^{3}}$ is a cube (since the map $c \mapsto c^{3}$ is a homomorphism from $\mathbb{F}_{Q^{3}}^{*}$ to itself which has trivial kernel). Thus the result holds in this case, so for the rest of this proof we will assume that $Q$ is a power of 4 . Let $\omega$ be a fixed primitive cube root of unity in $\mathbb{F}_{Q}$.

Pick $u, v \in \mathbb{F}_{Q^{3}}^{*}$ such that $u^{Q-1}+v^{Q-1}=1$. Write $U:=u^{Q-1}$ and $V:=v^{Q-1}$, so that $U+V=1$ and both $U$ and $V$ have order dividing $Q^{2}+Q+1$. We must show that $u v$ is a cube, or equivalently that $U V$ is a $3(Q-1)$-th power in $\mathbb{F}_{Q^{3}}^{*}$.

We first dispense with the case that $U$ is in $\mathbb{F}_{Q}$. If $U \in \mathbb{F}_{Q}$ then $V=U+1$ is also in $\mathbb{F}_{Q}$, so both $U$ and $V$ have order dividing $Q-1$. Thus the orders of $U$ and $V$ divide $Q^{2}+Q+1-(Q-1)(Q+2)=3$, so $U$ and $V$ are cube roots 
of unity whose sum is 1 . It follows that $U$ and $V$ are distinct primitive cube roots of unity, so their product is 1 , which is indeed a $3(Q-1)$-th power.

Henceforth we assume that neither $U$ nor $V$ is in $\mathbb{F}_{Q}$. Let $F(x)$ be the minimal polynomial of $U$ over $\mathbb{F}_{Q}$, so $F(x)=(x+U)\left(x+U^{Q}\right)\left(x+U^{Q^{2}}\right)$. Then $F(0)=U^{Q^{2}+Q+1}=1$. Since $F(x+1)$ is a monic irreducible polynomial in $\mathbb{F}_{Q}[x]$ which has $V$ as a root, it must be the minimal polynomial of $V$ over $\mathbb{F}_{Q}$. Its constant term $F(1)$ equals $V^{Q^{2}+Q+1}=1$. Therefore $F(x)+1$ is a monic degree-3 polynomial in $\mathbb{F}_{Q}[x]$ whose roots include 0 and 1 , so $F(x)+1=x(x+1)(x+b)$ for some $b \in \mathbb{F}_{Q}$.

Next we determine the minimal polynomial of $U V$ over $\mathbb{F}_{Q}$. Note that $U V=U^{2}+U$. Since $U$ has degree 3 over $\mathbb{F}_{Q}$, we must have $U^{2}+U \notin \mathbb{F}_{Q}$, so that $U^{2}+U$ also has degree 3 over $\mathbb{F}_{Q}$. Thus, the minimal polynomial of $U V$ over $\mathbb{F}_{Q}$ is the unique monic degree-3 polynomial in $\mathbb{F}_{Q}[x]$ which has $U V$ as a root. This polynomial is $G(x):=x^{3}+\left(b^{2}+b\right) x^{2}+x+1$, since

$$
\begin{aligned}
G\left(U^{2}+U\right) & =\left(U^{2}+U\right)^{3}+\left(b^{2}+b\right)\left(U^{2}+U\right)^{2}+U^{2}+U+1 \\
& =U^{6}+U^{5}+U^{4}\left(b^{2}+b+1\right)+U^{3}+U^{2}\left(b^{2}+b+1\right)+U+1 \\
& =(U(U+1)(U+b)+1) \cdot\left(U^{3}+b U^{2}+(b+1) U+1\right) \\
& =F(U) \cdot\left(U^{3}+b U^{2}+(b+1) U+1\right) \\
& =0 .
\end{aligned}
$$

Since $G(x)$ is the minimal polynomial of $U V$ over $\mathbb{F}_{Q}$, in particular it is irreducible. Thus $G(1) \neq 0$, so $b \notin\left\{\omega, \omega^{2}\right\}$. We will write down the minimal polynomials for all of the cube roots of $U V$. We first give three factorizations which might involve polynomials over an extension of $\mathbb{F}_{Q}$ :

$$
\begin{aligned}
G\left(x^{3}\right) & =\prod_{e^{3}=b^{2}+b+1}\left(x^{3}+e x^{2}+1\right) \\
& =\prod_{e^{3}=b+\omega}\left(x^{3}+e^{2} x^{2}+e x+1\right) \\
& =\prod_{e^{3}=b+\omega^{2}}\left(x^{3}+e^{2} x^{2}+e x+1\right) .
\end{aligned}
$$

We leave the easy verification of these factorizations to the reader; Magma code to verify them is contained in a comment in the TeX file. First suppose that one of these three factorizations only involves polynomials in $\mathbb{F}_{Q}[x]$. Since the cubes of the roots of $G\left(x^{3}\right)$ have degree 3 over $\mathbb{F}_{Q}$, it follows that the degree- 3 polynomials in this factorization are irreducible, so that any root $d$ of any of these degree-3 polynomials must satisfy $d^{Q^{2}+Q+1}=1$, and hence must be a $(Q-1)$-th power in $\mathbb{F}_{Q^{3}}^{*}$. Since $G(U V)=0$, this implies that $U V$ is a $3(Q-1)$-th power, as desired.

It remains to show that one of the three factorizations above only involves polynomials in $\mathbb{F}_{Q}[x]$. Equivalently, we must show that one of $b+\omega, b+\omega^{2}$, 
and $b^{2}+b+1$ is a cube in $\mathbb{F}_{Q}^{*}$. If this did not happen then, since $b^{2}+b+1=$ $(b+\omega)\left(b+\omega^{2}\right)$, the only possibility is that $b+\omega$ and $b+\omega^{2}$ are in the same coset in $\mathbb{F}_{Q}^{*} /\left(\mathbb{F}_{Q}^{*}\right)^{3}$. But then $\left(b+\omega^{2}\right) /(b+\omega)$ would be in $\left(\mathbb{F}_{Q}^{*}\right)^{3}$, so we could write $\left(b+\omega^{2}\right) /(b+\omega)=e^{3}$ with $e \in \mathbb{F}_{Q}^{*}$. Now one can easily verify that $d:=\left(b^{2}+b+1\right) e^{2}+(b+\omega)^{2} e+b^{2}+b$ is a root of $G(x)$ in $\mathbb{F}_{Q}$ (for instance, see the Magma code in the TeX file), which is impossible.

Next we prove a lemma expressing the planarity condition for monomials in a convenient form.

Lemma 2.1. Pick any positive integer $t$ and any $a \in \mathbb{F}_{q}^{*}$, where $q>1$ is a power of 2. Then $c \mapsto a c^{t}$ is a planar function on $\mathbb{F}_{q}$ if and only if, for each $b \in \mathbb{F}_{q}^{*}$, the function $c \mapsto(c+1)^{t}+c^{t}+c a^{-1} b^{t-2}$ is bijective on $\mathbb{F}_{q}$.

Proof. Planarity asserts that, for each $d \in \mathbb{F}_{q}^{*}$, the function $F: c \mapsto a(c+$ $d)^{t}+a c^{t}+d c$ is bijective on $\mathbb{F}_{q}$. Equivalently, $\ell_{1} \circ f \circ \ell_{2}$ is bijective, where $\ell_{1}: c \mapsto c /\left(a d^{t}\right)$ and $\ell_{2}: c \mapsto d c$. Since $\ell_{1} \circ f \circ \ell_{2}: c \mapsto(c+1)^{t}+c^{t}+c /\left(a d^{t-2}\right)$, putting $b=1 / d$ yields the result.

We now prove Theorem 1.1 .

Proof of Theorem 1.1. Let $k$ be a positive integer, and write $Q=4^{k}$ and $q=Q^{3}$. Let $a \in \mathbb{F}_{q}^{*}$ be a $(Q-1)$-th power which is not a $3(Q-1)$-th power.

We first show that no $d, e \in \mathbb{F}_{q}^{*}$ satisfy $d^{Q^{2}-1}+d^{Q-1}=a^{-1} e^{3 Q-3}$. For, suppose $d, e \in \mathbb{F}_{q}^{*}$ satisfy this equation. Since $a$ is a $(Q-1)$-th power, also $d^{Q^{2}-1}+d^{Q-1}$ is a $(Q-1)$-th power in $\mathbb{F}_{q}^{*}$. But $d^{Q^{2}-1}+d^{Q-1}=d^{Q-1}\left(d^{Q-1}+\right.$ $1)^{Q}$, so we must have $d^{Q-1}+1=u^{Q-1}$ for some $u \in \mathbb{F}_{q}^{*}$. Now Theorem 1.2 implies that $d u$ is a cube in $\mathbb{F}_{q}^{*}$, so $d^{Q-1} u^{Q^{2}-Q}=(d u)^{Q-1}\left(u^{(Q-1) / 3}\right)^{3 Q-3}$ is a $3(Q-1)$-th power; but this expression equals $a^{-1} e^{3 Q-3}$, which contradicts our hypothesis that $a$ is not a $3(Q-1)$-th power.

Thus, for each $e \in \mathbb{F}_{q}^{*}$, the polynomial $x^{Q^{2}-1}+x^{Q-1}+a^{-1} e^{3 Q-3}$ has no roots in $\mathbb{F}_{q}^{*}$. Since the function $c \mapsto c^{Q^{2}}+c^{Q}+c a^{-1} e^{3 Q-3}$ is a homomorphism from the additive group of $\mathbb{F}_{q}$ to itself, and the kernel of this homomorphism is trivial, the function is a bijection on $\mathbb{F}_{q}$. Since $(c+1)^{Q^{2}+Q}+c^{Q^{2}+Q}=$ $c^{Q^{2}}+c^{Q}+1$, this implies that $c \mapsto(c+1)^{Q^{2}+Q}+c^{Q^{2}+Q}+c a^{-1} e^{3 Q-3}$ is a bijection on $\mathbb{F}_{q}$. For any $b \in \mathbb{F}_{q}^{*}$, put $e=b^{(Q+2) / 3}$, so that $e^{3 Q-3}=b^{Q^{2}+Q-2}$. We have shown that $c \mapsto(c+1)^{Q^{2}+Q}+c^{Q^{2}+Q}+c a^{-1} b^{Q^{2}+Q-2}$ is a bijection on $\mathbb{F}_{q}$, which by Lemma 2.1 implies that $c \mapsto a c^{Q^{2}+Q}$ is planar on $\mathbb{F}_{q}$.

\section{Planar monomials of Degree $2^{i}+2^{j}$}

We conclude this paper with some general remarks about planar polynomials in characteristic 2 , and in particular about planar monomials of degree $2^{i}+2^{j}$. Here we say that $F(x) \in \mathbb{F}_{q}[x]$ is planar if the function $c \mapsto F(c)$ is a planar function on $\mathbb{F}_{q}$. Since every function $\mathbb{F}_{q} \rightarrow \mathbb{F}_{q}$ is represented by 
a polynomial, in particular every planar function is represented by a planar polynomial. Moreover, if $F, G \in \mathbb{F}_{q}[x]$ satisfy $F(x) \equiv G(x)\left(\bmod x^{q}-x\right)$ then $F(x)$ is planar if and only if $G(x)$ is planar. Thus, in order to classify planar polynomials, it suffices to classify their residues mod $\left(x^{q}-x\right)$, or equivalently, to classify planar polynomials of degree less than $q$.

If $p$ is any (odd or even) prime other than 3, then (for every $r$ ) all known planar polynomials over $\mathbb{F}_{p^{r}}$ of degree less than $p^{r}$ have the property that the degree of every term is the sum of at most two powers of $p$. The prime $p=3$ must be excluded, due to examples from [3]. Planar polynomials in which every term has degree the sum of at most two powers of $p$ are especially interesting, since a classification of such polynomials would be equivalent to a classification of finite commutative semifields [2]. To date, there is no $p$ for which there is even a conjectured classification of such planar polynomials. However, if we restrict to monomials then the situation becomes more tractable. It is easy to see that a monomial of degree $p^{i}$ is planar over $\mathbb{F}_{p^{r}}$ if and only if $p=2$. Further, it is well-known (and easy to prove) that, for any odd prime $p$ and any $0 \leq i \leq j<r$, a monomial over $\mathbb{F}_{p^{r}}$ of degree $p^{i}+p^{j}$ is planar if and only if $r / \operatorname{gcd}(r, j-i)$ is odd. Our Theorem 1.1 shows that the analogous assertion for $p=2$ is not true, and the complexity of our proof suggests that a classification of planar monomials over $\mathbb{F}_{2^{r}}$ of degree $2^{i}+2^{j}$ will likely be difficult to obtain. Here we make some remarks about this classification.

We first reformulate the planarity condition for these monomials:

Lemma 3.1. Fix $0 \leq i<j<r$ and $a \in \mathbb{F}_{2^{r}}^{*}$, and put $q=2^{r}$ and $G(x):=$ $x^{2^{i}-1}+x^{2^{j}-1}$. The monomial ax $x^{2^{i}+2^{j}}$ is planar on $\mathbb{F}_{q}$ if and only if the sets $G\left(\mathbb{F}_{q}^{*}\right)$ and $a^{-1}\left(\mathbb{F}_{q}^{*}\right)^{t-2}$ are disjoint.

Proof. Write $q=2^{r}$ and $t=2^{i}+2^{j}$. By Lemma 2.1, planarity asserts that, for each $b \in \mathbb{F}_{q}^{*}$, the polynomial $F(x):=(x+1)^{t}+x^{t}+x a^{-1} b^{t-2}$ induces a bijection on $\mathbb{F}_{q}$. Equivalently, $F(x)+1$ induces a bijection on $\mathbb{F}_{q}$, and we compute

$$
F(x)+1=x^{2^{i}}+x^{2^{j}}+x a^{-1} b^{t-2} .
$$

Since this polynomial induces a homomorphism from the additive group of $\mathbb{F}_{q}$ to itself, it induces a bijection if and only if it has no nonzero roots in $\mathbb{F}_{q}$. Since $F(x)+1=x\left(G(x)+a^{-1} b^{t-2}\right)$, the result follows.

Next we determine all planar monomials of degree $1+2^{j}$ :

Proposition 3.2. Pick $0<j<r$ and $a \in \mathbb{F}_{2^{r}}^{*}$, and let $T(x):=x^{2^{j-1}}+$ $x^{2^{j-2}}+\cdots+x$. Then ax $x^{1+2^{j}}$ is planar on $\mathbb{F}_{2^{r}}$ if and only if $j=r / 2$ and $T\left(a^{2^{j}+1}\right)=0$.

Proof. Let $q=2^{r}$ and $J=\operatorname{gcd}(j, r)$. By Lemma 3.1, $a x^{1+2^{j}}$ is planar on $\mathbb{F}_{q}$ if and only if there do not exist $u, v \in \mathbb{F}_{q}^{*}$ such that

$$
1+u^{2^{j}-1}=a^{-1} v^{2^{j}-1} .
$$


Since the set of $\left(2^{j}-1\right)$-th powers in $\mathbb{F}_{q}^{*}$ is the same as the set of $\operatorname{gcd}\left(2^{j}-\right.$ $\left.1,2^{r}-1\right)$-th powers, i.e., the $\left(2^{J}-1\right)$-th powers, planarity asserts that the equation $1+U^{2^{J}-1}=a^{-1} V^{2^{J}-1}$ has no solutions with $U, V \in \mathbb{F}_{q}^{*}$. Note that the equation $z^{2^{J}-1}+x^{2^{J}-1}=a^{-1} y^{2^{J}-1}$ defines a nonsingular curve $C$ in $\mathbb{P}^{2}$ of genus $g:=\left(2^{J}-2\right)\left(2^{J}-3\right) / 2$. Planarity asserts that $C$ has no $\mathbb{F}_{q^{-} \text {-rational }}$ points with nonzero coordinates.

First suppose $J \leq r / 4$. Then Weil's bound implies that the number of $\mathbb{F}_{q}$-rational points on $C$ is at least

$$
\begin{aligned}
q+1-2 g \sqrt{q} & =q+1-\left(2^{J}-2\right)\left(2^{J}-3\right) \sqrt{q} \\
& \geq q+1-\left(q^{1 / 4}-2\right)\left(q^{1 / 4}-3\right) \sqrt{q} \\
& =1+\left(5 q^{1 / 4}-6\right) \sqrt{q} .
\end{aligned}
$$

At most $3\left(2^{J}-1\right)$ of these points have a coordinate being zero. Since

$$
1+\left(5 q^{1 / 4}-6\right) \sqrt{q}>3 \sqrt{q}>3\left(2^{J}-1\right)
$$

there is a point in $C\left(\mathbb{F}_{q}\right)$ with nonzero coordinates, so $a x^{1+2^{j}}$ is not planar on $\mathbb{F}_{q}$.

Since $J$ is a proper divisor of $r$, the remaining cases are $J=r / 2$ and $J=$ $r / 3$. If $J=r / 2$ then $j=r / 2$, and by [6, Thm. 1], there is a point in $C\left(\mathbb{F}_{q}\right)$ with nonzero coordinates if and only if the canonical additive character $\chi$ on $\mathbb{F}_{2^{J}}$ satisfies $\chi\left(a^{2^{J}+1}\right) \neq 1$, or equivalently $T\left(a^{2^{J}+1}\right) \neq 0$. If $J=r / 3$ then, by [6, Thm. 2], the curve $C$ has an $\mathbb{F}_{q}$-rational point with nonzero coordinates.

Remark. If $j=r / 2$ then the condition $T\left(a^{2^{j}+1}\right)=0$ can be reformulated as asserting that $a^{2^{j}+1}=b^{2}+b$ for some $b \in \mathbb{F}_{2^{j}}$. The number of elements $a \in \mathbb{F}_{2^{r}}^{*}$ which satisfy this condition is $\left(2^{j-1}-1\right)\left(2^{j}+1\right)$. Among these are precisely $2^{j-1}-1$ elements of $\mathbb{F}_{2^{j}}^{*}$, namely, the elements of the form $b^{2}+b$ with $b \in \mathbb{F}_{2^{j}} \backslash \mathbb{F}_{2}$. Planarity of $a x^{1+2^{j}}$ for these latter $a$ 's was shown in [8, Thm. 6].

Since the $(t-2)$-th powers in $\mathbb{F}_{q}^{*}$ are precisely the $\operatorname{gcd}(t-2, q-1)$-th powers, Lemma 3.1 implies that $a x^{2^{i}+2^{j}}$ cannot be planar on $\mathbb{F}_{q}$ if $\operatorname{gcd}\left(2^{i}+\right.$ $\left.2^{j}-2, q-1\right)=1$. This is a special case of [8, Prop. 9]. It follows that there are no planar monomials over $\mathbb{F}_{2^{r}}$ of degree $2+2^{j}$ if $1<j<r$. But we have not gotten much further in determining which monomials of degree $2^{i}+2^{j}$ are planar. In particular, we do not know whether there are any other planar monomials over $\mathbb{F}_{Q^{3}}$ of degree $Q+Q^{2}$, besides the ones described in Theorem 1.1. However, we did verify via computer that the planar monomials in Theorem 1.1 and Proposition 3.2 are the only planar monomials over $\mathbb{F}_{2^{r}}$ of degree $2^{i}+2^{j}$ with $0 \leq i<j<r \leq 50$. 


\section{REFERENCES}

[1] C. Carlet, C. Ding and J. Yuan, Linear codes from perfect nonlinear mappings and their secret sharing schemes, IEEE Trans. Inform. Theory 51 (2005), 2089-2102.

[2] R. S. Coulter and M. Henderson, Commutative presemifields and semifields, Adv. Math. 217 (2008), 282-304.

[3] R. S. Coulter and R. W. Matthews, Planar functions and planes of Lenz-Barlotti class II, Des. Codes Cryptogr. 10 (1997), 167-184.

[4] P. Dembowski and T. G. Ostrom, Planes of order $n$ with collineation groups of order $n^{2}$, Math. Z. 103 (1968), 239-258.

[5] M. J. Ganley and E. Spence, Relative difference sets and quasiregular collineation groups, J. Combin. Theory Ser. A 19 (1975), 134-153.

[6] M. Moisio, On the number of rational points on some families of Fermat curves over finite fields, Finite Fields Appl. 13 (2007), 546-562.

[7] K. Nyberg and L. R. Knudsen, Provable security against differential cryptanalysis, in: Advances in Cryptology (CRYPTO '92), Lecture Notes in Computer Science 740, Springer-Verlag (1992), 566-574.

[8] K.-U. Schmidt and Y. Zhou, Planar functions over fields of characteristic two, J. Algebraic Combin. 40 (2014), 503-526.

[9] J. F. Voloch and M. E. Zieve, Rational points on some Fermat curves and surfaces over finite fields, Int. J. Number Theory 10 (2014), 319-325.

[10] Y. Zhou, $\left(2^{n}, 2^{n}, 2^{n}, 1\right)$-relative difference sets and their representations, J. Combin. Des. 21 (2013), 563-584.

Department of Mathematics, University of Pennsylvania, 209 South 33rd

Street, Philadelphia, PA 19104-6395 USA

E-mail address: zscherr@math.upenn.edu

URL: http://www. math.upenn.edu/ zscherr/

Department of Mathematics, University of Michigan, 530 Church Street, AnN ARBor, MI 48109-1043 USA

Mathematical Sciences Center, Tsinghua University, Beijing 100084, China

E-mail address: zieve@umich.edu

$U R L:$ http://www.math.Isa.umich.edu/ zieve/ 